ACCEPTED MANUSCRIPT

\title{
Status and prospects of ohmic contacts on two dimensional semiconductors
}

To cite this article before publication: Junhao Ni et al 2021 Nanotechnology in press https://doi.org/10.1088/1361-6528/ac2fe1

\section{Manuscript version: Accepted Manuscript}

Accepted Manuscript is "the version of the article accepted for publication including all changes made as a result of the peer review process, and which may also include the addition to the article by IOP Publishing of a header, an article ID, a cover sheet and/or an 'Accepted Manuscript' watermark, but excluding any other editing, typesetting or other changes made by IOP Publishing and/or its licensors"

This Accepted Manuscript is @ 2021 IOP Publishing Ltd.

During the embargo period (the 12 month period from the publication of the Version of Record of this article), the Accepted Manuscript is fully protected by copyright and cannot be reused or reposted elsewhere.

As the Version of Record of this article is going to be / has been published on a subscription basis, this Accepted Manuscript is available for reuse under a CC BY-NC-ND 3.0 licence after the 12 month embargo period.

After the embargo period, everyone is permitted to use copy and redistribute this article for non-commercial purposes only, provided that they adhere to all the terms of the licence https://creativecommons.org/licences/by-nc-nd/3.0

Although reasonable endeavours have been taken to obtain all necessary permissions from third parties to include their copyrighted content within this article, their full citation and copyright line may not be present in this Accepted Manuscript version. Before using any content from this article, please refer to the Version of Record on IOPscience once published for full citation and copyright details, as permissions will likely be required. All third party content is fully copyright protected, unless specifically stated otherwise in the figure caption in the Version of Record.

View the article online for updates and enhancements. 


\title{
Status and Prospects of Ohmic Contacts on Two Dimensional Semiconductors
}

\author{
Junhao $\mathrm{Ni}^{\mathrm{a}, \#}$, Quangui Fu ${ }^{\mathrm{a}, \#}$, Kostya (Ken) Ostrikov ${ }^{\mathrm{b}}$, Xiaofeng Gu, Haiyan Nan ${ }^{\mathrm{a},{ }^{*} \text {, and }}$ \\ Shaoqing Xiao ${ }^{\mathrm{a}, *}$
}

${ }^{a}$ Engineering Research Center of IoT Technology Applications (Ministry of Education), Department of Electronic Engineering, Jiangnan University, Wuxi 214122, China.

${ }^{\mathrm{b}}$ School of Chemistry and Physics and QUT Centre for Materials Science, Queensland University of Technology (QUT), Brisbane QLD 4000, Australia

*Corresponding author: jnanhaiyan@jiangnan.edu.cn (Haiyan Nan) xiaosq@jiangnan.edu.cn (Shaoqing Xiao)

\#These authors contributed equally.

\begin{abstract}
In recent years, two-dimensional materials have received more and more attention in the development of semiconductor devices, and their practical applications in optoelectronic devices have also developed rapidly.However, there are still some factors that limit the performance of two-dimensional semiconductor material devices, and one of the most important is Ohmic contact. Here, we elaborate on a variety of approaches to achieve Ohmic contacts on two dimensional materials and reveal their physical mechanisms. For the work function mismatch problem, we summarize the comparison of barrier heights between different metals and 2D semiconductors. We also examine different methods to solve the problem of Fermi level pinning. For the novel 2D metalsemiconductor contact methods, we analyse their effects on reducing contact resistance from two different perspectives: homojunction and heterojunction. Finally, the challenges of 2D semiconductors in achieving Ohmic contacts are outlined.
\end{abstract}

Keywords: two-dimensional materials; Ohmic contact; work function; Schottky barrier; Fermi level pinning; transferring electrode; 2D metal-semiconductor contact

\section{Introduction}

In the past half century, the maturity of complementary metal oxide semiconductor (CMOS) field effect transistor (FET) devices based on silicon (Si) has enabled modern information technology to develop by leaps and bounds [1,2]. According to Moore's Law, the integration density of FETs on a chip can be doubled and the feature size of FETs can be shrinked greatly in every two years [3]. When the channel length is close to a few nanometers, problems such as short channel effect and high heat dissipation will emerge, indicating that traditional siliconbased devices are approaching their limits. Therefore, it is urgent to explore new alternative materials [4-6]. In this context, 2D semiconductor materials such as graphene [7], transition metal dichalcogenides (TMDC) [8-12], Black Phosphorous (BP) [13, 14], etc. have received more and more attention in recent years. In particular, Ajayan et al. has made a comprehensive summary on the characteristics, preparation and application of different 2D semiconductor matericals [15]. The 2D materials are so atomically thin that the channel length of FETs based on such materials can be further reduced without short channel effect. Moreover, due to their unsuspended surface, the devices based on such materials can greatly eliminate carrier scattering, representing an obvious advantage over traditional materials like Si [16-20]. These excellent features of 2D materials have made them promising for a variety of electronic and optoelectronic devices including field effect transistors [21], photodetectors [22], logic devices [23], light-emitting diodes [24] etc. 
The contacts between metallic electrodes and semiconductor materials are crucial components of electronic and optoelectronic devices [25], especially for 2D materials that are always accompanied by 3D metallic electrodes. In fact, contacts are the communication bridge between 2D materials and the three-dimensional world [26]. Such contacts are either Ohmic contacts or Schottky junctions. The latter may result in a huge contact resistance and thus hinder the transport of carriers [27, 28], degrade the transconductance [29], switching current ratio and other properties. Therefore, for most devices, Ohmic contact is a guarantee of high performance [26, 30-32]. To this end, researchers have explored many new methods in recent years to achieve Ohmic contacts or reduce 2D contact resistance, such as one-dimensional contact [33-36]; phase engineering [37-39]; electrode transfer [40] and so on. Precise engineering of ohmic contacts in two-dimensional materials is rapidly gaining momentum as summarized in a recent review [41]. This article complements the analysis of the recent advances in this area by examining the effective approaches to fabricate Ohmic contacts on 2D materials as well as the relevant physical mechanisms.

In this review, we will elaborate on the latest research progress of different methods in achieving Ohmic contacts or reducing contact resistances for 2D materials devices. They can be mainly classified into three ones: using metal electrodes with matched work function, solving the problem of Fermi pinning level and using 2D metalsemiconductor contacts. We first examine the work function matching between the most widely studied 2D materials like graphene, TMDCs and BP and different metals, and pinpoint the most suitable metals for these 2D materials in achieving Ohmic contacts. We then study the problem of Fermi level pinning and reveal the physical mechanism. Through different contact engineering, the Fermi level pinning effect at the interface can be weakened and even almost completely suppressed. Finally, in the third part, we shift our sight from 3D to 2D, and focus on effective methods for 2D metal materials to reduce contact resistance from the perspective of homojunction and heterojunction. The main motivation of this review is to establish the associations between different contact methods and the properties of $2 \mathrm{D}$ semiconductors to facilitate our understandings toward how to realize Ohmic contacts on 2D semiconductors and improve the performance of their devices.

\section{Using metal electrodes with matched work function}

In theory, the contact resistance mainly depends on the height of the Schottky barrier. When the metal electrode and the semiconductor interface are in contact, due to the difference between the metal work function and the semiconductor affinity (ionization potential), the energy band will be bent and the Schottky barrier will be generated. Under ideal circumstances, according to Schottky-Mott's law [42, 43]:

$$
\begin{gathered}
\Phi_{B, n}=\phi_{M}-\chi_{S C} \\
\Phi_{B, p}=I_{S C}-\phi_{M}
\end{gathered}
$$

where $\Phi_{B, n}$ and $\Phi_{B, p}$ are the Schottky barrier heights for electron and hole injection respectively, $\phi_{M}$ is the metal work function, $\chi_{S C}$ and $I_{S C}$ are the electron affinity and ionization potentials respectively. In theory, we can minimize the height of the Schottky barrier by selecting a suitable metal electrode to reduce the contact resistance. The minimum of the Schottky barrier is zero corresponding to Ohmic contact. Therefore, we will systematically study the work function matching between different metals and the most widely studied 2D materials like graphene, TMDC (mainly $\mathrm{MoS}_{2}$ ), and BP in achieving Ohmic contacts in this section.

\subsection{Graphene}

Unlike other 2D materials, graphene has a zero-band gap, so it doesn't form a depletion layer and traditional Schottky barrier when it contacts with metal. The contact between metals with different work functions and graphene will cause graphene to be electrically doped, causing the Fermi level of graphene to move away from the Dirac point. Although graphene has good conductivity, the small density of states near the Dirac point limits the carrier transfer efficiency between metal and graphene, resulting in high contact resistance in the contact area [30]. By using the transmission line model measurement method, Osman et al. measured the contact resistance of Au and graphene to be $630 \Omega \cdot \mu \mathrm{m}, \mathrm{Ag}-630 \Omega \cdot \mu \mathrm{m}, \mathrm{Cu}-8800 \Omega \cdot \mu \mathrm{m}, \mathrm{Pd}-570 \Omega \cdot \mu \mathrm{m}$ [44] and Ni-2200 $\Omega \cdot \mu \mathrm{m}$ [45]. It is obvious that the contact resistance of $\mathrm{Pd}$ and $\mathrm{Au}$ is the smallest and this is why we often use Au as the metal electrodes in daily experiments. In addition to a single metal electrode, a combination of multiple metals is generally employed in daily experiments. For this reason, the contact resistance of a combination of multiple metals in contact with graphene is also listed. Au is the most widely used choice among the combined metals. When the previous single metal and $\mathrm{Au}$ are combined to server as the contact, the contact resistance drops significantly. The contact resistance of $\mathrm{Cu} / \mathrm{Au}$ drops to $92 \Omega \cdot \mu \mathrm{m}$ [46], which is reduced by two orders of magnitude 
$\mathrm{Pd} / \mathrm{Au}-122 \Omega \cdot \mu \mathrm{m}[46] ; \mathrm{Ni} / \mathrm{Au}-400 \Omega \cdot \mu \mathrm{m}$ [45]; Ti/Au- 23 $\Omega \cdot \mu \mathrm{m}$ [46]. It is clear that Ti/Au and Cu/Au mixed contacts are the most appropriate for graphene in reducing the contact resistance.

\subsection{TMDCs and BP}

As a typical n-type representative semiconductor of TMDCs, $\mathrm{MoS}_{2}$ has received extensive attention due to its excellent performance. Here, we mainly study the Schottky barrier height when $\mathrm{MoS}_{2}$ is in contact with metals with different work functions, and summarize the metal-semiconductor contacts closest to Ohmic contact. The left side of Figure 1 (a) is the energy band of $\mathrm{MoS}_{2}$ and the work function arrangement of commonly used metals, and the right side is the n-type Schottky barrier height where different metals contact with $\mathrm{MoS}_{2}$. Among them, the red part is the electronic Schottky barrier height obtained through theoretical calculation [9], and the blue part is the Schottky barrier height extracted in the actual experiment [11, 47, 48]. It can be found that the Fermi levels of Sc and $\mathrm{Ti}$ are closer to the conduction band of $\mathrm{MoS}_{2}$, and the Schottky barrier heights between Sc and $\mathrm{MoS}_{2}$ as well as between $\mathrm{Ti}$ and $\mathrm{MoS}_{2}$ are the smallest. Comparing theoretically calculated barrier heights with actual ones for all the metals, one can find a large difference between them except the metals including Au and Pt. This means that $\mathrm{Au}$ and Pt metal electrodes are very hardly affected by the surface states. In addition to Sc and Ti, Kang et al. have also studied metal Mo as an electrode in contact with $\mathrm{MoS}_{2}$. Because Mo is the constituent metal of the semiconductor material, strong atomic orbital overlap occurs at the contact interface, thus giving rise to a smaller contact resistance than Ti. As show in Figure 1(b), the SBH of Mo-MoS 2 system is much less than Ti-MoS 2 system $[49,50]$. In addition, Wang et al. have achieved van der Waals contacts (as shown in Figure 1(c)) to reduce contact resistance by evaporating $10 \mathrm{~nm}$ thick In between $100 \mathrm{~nm}$ thick Au and a single layer of $\mathrm{MoS}_{2}$ [51]. The experimentally extracted Schottky barrier is about $110 \mathrm{meV}$, and the contact resistance on the single-layer $\mathrm{MoS}_{2}$ is measured to be about $3 \mathrm{k} \Omega \mu \mathrm{m}$, and that on the multilayer $\mathrm{MoS}_{2}$ is $0.8 \mathrm{k} \Omega \mu \mathrm{m}$, which is one of the smallest values so far. This resulted in a huge increase in mobility (Figure 1(c)) [51]. In brief, in addition to Sc and Ti with low work function, the special metals Mo and In can also achieye almost Ohmic contact for $\mathrm{MoS}_{2}$. Similarly, for another n-type TMDCs like $\mathrm{WS}_{2}$, the most appropriate metals to achieve Ohmic contact are Sc, Ti, W and In [49].

However, for typical p-type TMDCs like $\mathrm{WSe}_{2}$, the metals with high work function are preferred instead to achieve Ohmic contact. As shown in Figure 1(d), the Schottky barrier heights of holes between WSe $\mathrm{W}_{2}$ and different metals are 1.27, 1.25, 1.13, 0.9 and $0.35 \mathrm{eV}$, corresponding to Ti, W, In, Au, and Pd. Therefore, the most appropriate metals to achieve Ohmic contact for $\mathrm{WSe}_{2}$ is $\mathrm{Pd}$.

BP shows great potential in 2D electronic and optoelectronic devices because of its high mobility and direct band gap. Few-layer BP flakes with 3-15 layers are often used in practice, which possess a bandgap ranging from 0.3 to $0.6 \mathrm{eV}$. The band gap can be calculated theoretically: $E_{\text {gap }}=0.39 \mathrm{eV}+1.62 / \mathrm{n}^{1.4}$ (n=number of layers). As the number of BP layers increases, the band gap decreases. In order to study the influence of different metals and $\mathrm{BP}$ contact, Wang et al. listed the Schottky barrier heights extracted from five different metals Ti, Ni, Ag, Sc, Er in contact with BP under the alumina package [52] (Figure1(e)). It can be seen from the figure that Ni has the lowest hole barrier, which is more conducive to hole injection, so as to achieve high-quality p-type contact, which is also proved by experimental results. Zhi-peng et al. reported that when using Ni in contact with $12 \mathrm{~nm}$ thick BP, the extracted Schottky barrier is $92 \mathrm{meV}$; when using $15 \mathrm{~nm}$ BP in contact with $\mathrm{Ni}, \mathrm{Ni}_{2} \mathrm{P}$ is formed after annealing at $300^{\circ} \mathrm{C}$, making the contact potential barrier reduced to $12 \mathrm{meV}$ [53]. Compared with low work function metals $\mathrm{Co}$ and $\mathrm{Ti}$ as contact metals (extracted Schottky barriers are $206 \mathrm{meV}$ [54] and 325meV [55], respectively), Ni shows obvious advantages. In addition to Ni, high work function metal Pd is also a common metal. Yuqiang Ma et al. synthesized $\mathrm{Pd}-\mathrm{H}$ alloy by reacting with $\mathrm{H}_{2}$ to increase the work function of the metal, thereby reducing the barrier height and making the contact resistance from $7.1 \Omega \cdot \mathrm{mm}$ is reduced to $1.05 \Omega \cdot \mathrm{mm}$ [56]. As a supplement to $\mathrm{BP}$ performance, bipolar transport can be obtained by using $\mathrm{Al}\left(\phi_{\mathrm{Al}}=4.2 \mathrm{eV}\right)$ with $\mathrm{BP}$ contact, which provides more possibilities for the application of BP in CMOS [57, 58].

\section{Solving pinning effect}

Ideally, the height of the Schottky barrier between a metal and a semiconductor is determined by the metal work function, but in practice, the $\Phi_{\mathrm{B}}$ in formula (1) is usually insensitive to $\Phi_{\mathrm{M}}$. This is mainly due to the fact that the Fermi level of the system is typically pinned to a nearly fixed position in the semiconductor bandgap, varying little with respect to different metals used. The Schottky-Mott law should be rewritten as following when considering the Fermi pinning effect [59]:

$$
\Phi_{B}=\boldsymbol{S}\left(\phi_{M}-\phi_{C N L}\right)+\left(\phi_{C N L}-\chi\right)
$$


where $\mathrm{S}$ is the Schottky pinning coefficient $\left.\left(\mathrm{S}=d \phi_{B} / d \Phi_{M}\right)\right), \chi$ is the electron affinity of the semiconductor, and $\phi_{\mathrm{CNL}}$ is the charge intermediate performance level under a relative vacuum level. Generally, the Fermi level will be pinned near $\phi_{\mathrm{CNL}}$, it is given by the following formula:

$$
\phi_{C N L}=\frac{\chi+b}{1-S}
$$

where $\mathrm{b}$ is the y-intercept of the $\phi_{B}$ versus $\phi_{M}$ graph, $\mathrm{S}$ depends on the interface state density per unit area $\mathrm{N}$ and the length $\delta$ extending into the semiconductor as:

$$
S=\frac{1}{1+\frac{q^{2} N \delta}{\varepsilon_{0} \varepsilon_{r}}}
$$

where $\varepsilon_{0} \varepsilon_{r}$ is the absolute permittivity of the interface. When $\mathrm{S}$ is equal to 0 , strong Fermi level pinning effect dominates so that $\mathrm{SBH}$ has nothing to do with the work function of the metal; when $\mathrm{S}$ is close to 1 , the Barding limit converges to the Schottky limit.

The origin of Fermi level pinning may be mainly due to the existence of interface dipoles and metal-induced interstitial states (MIGS) [60]. The interface dipoles will change the metal work function, and the metal-induced interstitial states will weaken the layer Molybdenum-sulfur bond through the interaction between the metal and the chalcogenide element, thereby pinning the Fermi level [60]. In addition, the existence of defects is considered to be one of the main reasons for Fermi level pinning [61, 62]. The SBH difference between theoretical and experimental values as illustrated in Section 1.2 may be caused by the defect state, which is attributed to atomic vacancies and process-induced defects [63]. Studies on the pinning factor of the defect area and the original surface show that in most cases, the pinning factor of the defect area is observed to be about 30-40\% smaller than that of the original surface, which indicates that the FLP is stronger at the defect location [64]. Guo et al.'s study of Schottky barrier heights for a variety of metals showed that any defects formed at the interface will cause additional pinning and reduce the $\mathrm{S}$ value [65]. Therefore, improving the quality of the interface is essential for achieving high-performance Ohmic contacts.

\subsection{Reducing interface impact}

One of the main methods to alleviate the Fermi level pinning effect is to insert an ultra-thin oxide layer between the metal and the semiconductor to realize the MIS structure. The insertion of the oxide layer can isolate the direct contact between the metal and the 2D semiconductor, and thus suppress the Fermi level pinning effect by reducing the metal-induced interstitial state. Also, the insertion of the oxide layer can ensure the cleanness of the interface. Various metal oxides have been used as insulating layers to reduce the pinning effect, such as $\mathrm{MoO}_{\mathrm{x}}, \mathrm{MgO}, \mathrm{Ta}_{2} \mathrm{O}_{5}$, $\mathrm{TiO}_{2}$ and so on [66-69]. As early as 2013, Chen et al. found that by inserting $\mathrm{MgO}$ between Co and $\mathrm{MoS}_{2}$, the height of the Schottky barrier can be reduced by $84 \%$ [66]. In addition to $\mathrm{MgO}, \mathrm{TiO}_{2}$ is also a commonly used oxide. In 2014, Dankert et al. also used $\mathrm{Co}$ as a contact and changed the interface state by inserting a $\mathrm{TiO}_{2}$ insulating layer between Co and $\mathrm{MoS}_{2}$. As a result, the height of the extracted Schottky barrier was reduced from $121 \mathrm{meV}$ to $27 \mathrm{meV}$, and the on-state current of the transistor increased by two orders of magnitude, as shown in Figures 2(a) and 2(b) [67] Recently, Kim has further studied the reason why the oxide layer alleviates the Fermi level pinning effect by inserting $1 \mathrm{~nm} \mathrm{TiO}_{2}$ between various metals and $\mathrm{MoS}_{2}$. As shown in Figures 2(c) and 2 (d), the pinning coefficient $\mathrm{S}$ is increased from 0.02 to 0.24 after inserting $\mathrm{TiO}_{2}$, showing a stronger work function dependence. The Fermi level pinned in the intermediate state is moved more toward their ideal position through the reduced interface state of the oxide layer, thereby weakening the pinning effect [68]. Lee et al. reduced the barrier height from $95 \mathrm{meV}$ to $29 \mathrm{meV}$ by inserting $\mathrm{Ta}_{2} \mathrm{O}_{5}$ between $\mathrm{MoS}_{2}$ and metal contacts [69]. As shown in Figure 2(e), the authors believed that the MIS structure can alleviate the Fermi level pinning mainly due to two points: (1) The metal will make the semiconductor Fermi level move to the charge middle performance level, and the oxide layer can weaken this effect; (2) Interfacial dipoles will be formed on the interface of oxide and semiconductor, and the opposite electric field of the interfacial dipoles will also restrict the Fermi energy level to move to the charge middle performance level. At the same time, Lee et al. also found that the thickness of the oxide layer also has an effect on the barrier height. The barrier height will increase as the oxide layer thickness increases [69].

In addition to the oxide layer, inserting h-BN to form the MIS structure can also effectively suppress the Fermi level pinning effect $[70,71]$ with similar physical mechanisms. BN can help form a high-quality interface with 2D semiconductors to prevent the formation of defects and traps. Wang et al. reduced the barrier height from $158 \mathrm{meV}$ to $31 \mathrm{meV}$ and the contact resistance from $5.1 \mathrm{k} \Omega \mu \mathrm{m}$ to $1.8 \mathrm{k} \Omega \mu \mathrm{m}$ by inserting h-BN between $\mathrm{MoS}_{2}$ and Ni/Au [70]. In addition to intercalation, it is also a feasible method to reduce the Fermi level pinning effect by treating 
the metal surface. Min et al. weakened the Fermi level pinning effect by atomically passivating gold, reducing the barrier height to $0.12 \mathrm{eV}$, almost realizing Ohmic contact [72]. In addition, ultra-high vacuum deposition of metal electrodes can make the metal/semiconductor interface extremely clean and thus greatly reduce the contact resistance. For example, the contact resistance of $\mathrm{MoS}_{2} / \mathrm{Au}$ contact with the metal electrode deposited under ultrahigh vacuum conditions $\left(10^{-9}\right.$ Torr $)$ is three times lower than that under normal conditions $\left(10^{-6}\right.$ Torr $)$ [73]. Recently, Matkovic, et al. successfully adjusted the work function of the metal electrode by using a self-assembled monolayers(SAMs) containing pyrimidine, so that the contact resistance between $\mathrm{MoS}_{2}$ monolayer and metal electrode was reduced by two orders of magnitude. This work brings a new but well-understood approach in organic electronics to realize ohmic contact in the field of 2D materials [74].

\subsection{Transfering electrode}

The above-mentioned multiple methods can partially reduce the Fermi level pinning effect, while transferring electrode as proposed and demonstrated by Liu et al. can almost completely eliminate the Fermi level pinning effect [40]. By pre-fabricating metal electrodes and transferring them onto the two-dimensional semiconductor surface via physical lamination, related chemical barriers and defect-induced gap states are completely eliminated. As shown in Figures 3(a) and 3(b), compared to the metal/semiconductor interface prepared by traditional deposition method, that prepared by transferring electrode method has an atomically sharp and clean interface, without defects, strain, metal diffusion, etc. Physical contact without direct chemical bonding can greatly inhibit the generation of interstitial states induced by interface dipoles and metals, thereby eliminating Fermi level pinning. Figure 3(c) shows the transfer characteristic curves of $\mathrm{MoS}_{2}$ FETs with electron beam deposited metal electrodes. It can be seen that regardless of the different work function of the contact metals, all curves showntype behaviour. This indicates that there is a strong Fermi level pinning effect near the $\mathrm{MoS}_{2}$ conduction band. On the contrary, for the FETs with transferring metal electrodes, as shown in Figure 3(c), the transfer curves can exhibit different polarities with the change of the work function of different metals. For low work function metal like Ag, a good n-type transfer curve is observed; as the work function of the metal increases, it shows a transition from n-type to bipolar and then to p-type. By using the extracted Schottky barrier height, the Fermi level pinning coefficient is fitted in Figure 3(d). For the FET devices with deposited metal, the extracted S parameter is 0.09, which is consistent with the previous results. For the devices with transferring electrode, the fitted $\mathrm{S}$ parameter is 0.96, which is approaching the Schottky-Mott limit, indicating that the height of the Schottky barrier depends dominantly on the metal work function.

\section{2D-semiconductor-metal contact}

Using 2D metal materials instead of traditional metals to contact 2D semiconductors is also a good strategy to eliminate the Fermi level pinning effect. Homogeneous structures or heterostructures formed by 2D metals and 2D semiconductors can strongly inhibit the formation of metal-induced interstitial states in semiconductors, thereby reducing the Fermi level pinning effect. In this section, we thus elaborate on effective methods for $2 \mathrm{D}$ metal materials to reduce contact resistance from the perspective of homojunction and heterojunction.

\subsection{Homojunction}

For TMDCs, in addition to the $2 \mathrm{H}$ phase semiconductor state, they also have $1 \mathrm{~T}$ and $1 \mathrm{~T}^{\prime}$ metal states. As shown in Figure 4(a), both $2 \mathrm{H}$ and $1 \mathrm{~T}$ (1 $\mathrm{T}^{\prime}$ ) phases possess the same lattice constant so that their in-plane connections are perfectly aligned without any interface defects [75, 76]. For example, as shown in Figure 4(b), it is effective to grow lateral homojunction consisting of $2 \mathrm{H} \mathrm{MoTe} \mathrm{H}_{2}$ and $1 \mathrm{~T} \mathrm{MoTe} \mathrm{H}_{2}$ by chemical vapor deposition via adjusting growth temperature since the energy difference between the $1 \mathrm{~T}$ phase and the $2 \mathrm{H}$ phase is very small ( $35 \mathrm{meV}$ per unit cell) $[77,78]$. Compared with the traditional contact consisting of $2 \mathrm{H}$ semiconducting phase $\mathrm{MoTe}_{2}$ and metal electrode where a Schottky contact is always formed, a novel contact consisting of $2 \mathrm{H} / 1 \mathrm{~T}$ ' homojunction and metal electrode is fabricated where a stable covalent bond is formed between the $1 \mathrm{~T}^{\prime}$ phase and the $2 \mathrm{H}$ phase. In this case, the $1 \mathrm{~T}$ ' metallic phase serves as the transition region between $2 \mathrm{H}$ semiconducting phase and metal electrode. As a result, the contact resistance is reduced significantly and the conductance of the $2 \mathrm{H}$ semiconducting channel is increased by two orders of magnitude. As shown in Figure 4(c), the height of the Schottky barrier is reduced greatly from $150 \mathrm{meV}$ for the traditional contact consisting of $2 \mathrm{H} \mathrm{MoTe} 2$ and metal electrode to $22 \mathrm{meV}$ for the novel contact consisting of $2 \mathrm{H} / 1 \mathrm{~T}$ ' homojunction and metal electrode. Such Chemical Vapor Deposition (CVD) method can also be applicable to the fabrication of $\mathrm{WSe}_{2} 2 \mathrm{H} / 1 \mathrm{~T}$ homojunction for reducing the Fermi level pining effect $[79,80]$. 
In addition, phase engineering of existing $2 \mathrm{H}$-phase TMDCs to transform them into metallic phases is also a commonly used method [37, 38, 81]. As early as 2014, kappera et al.[37] transformed the $2 \mathrm{H}$ phase of $\mathrm{MoS}_{2}$ into $1 \mathrm{~T}$ phase through a local induction method, as shown in Figure 4(d). The contact resistance measured under zero grid bias is only $200 \Omega \cdot \mu \mathrm{m}$, which is the lowest value ever for $\mathrm{MoS}_{2}$ [37]. Cho et al. also realized the transformation of $\mathrm{MoTe}_{2}$ from $2 \mathrm{H}$ phase to $1 \mathrm{~T}^{\prime}$ phase by laser burning method and systematically studied their Schottky barriers. The Schottky barrier height in the 1T' phase is only $10 \mathrm{meV}$ and the field-effect mobility is increased by 50 times [82], indicating that Ohmic contact is nearly realized.

Interestingly, for some specific 2D materials such as $\mathrm{PtSe}_{2}$, the phase state depends on the thickness of the material. The thick layer behaves as a metal state, while the few layers becomes a semiconductor state. In such case, homogeneous junction can be fabricated by superimposing two $\mathrm{PtSe}_{2}$ layers with different thicknesses. Indeed, Das et al. achieved a low-resistance Ohmic contact by transferring multiple layers of $\mathrm{PtSe}_{2}$ onto three layers of $\mathrm{PtSe}_{2}$ (Figure 4(e) [83]. The barrier height was reduced from $140 \mathrm{meV}$ for the traditional contact consisting of $3 \mathrm{~L} \mathrm{PtSe} e_{2}$ and metal electrode to $35 \mathrm{meV}$ for the novel contact consisting of $\mathrm{ML} \mathrm{PtSe}_{2} / 3 \mathrm{LtSe} \mathrm{Pt}_{2}$ and metal electrode [83].

\subsection{Heterojunction}

In addition to fabricating 2D metal/semiconductor homojunctions made of different phases of the same material to reduce the contact resistance, fabricating $2 \mathrm{D}$ metal/semiconductor heterojunctions made of different $2 \mathrm{D}$ materials is also an alternative choice. Graphene, as a metallic 2D material with adjustable Fermi level, is usually used as the intermediate metal layer [84, 85]. As shown in Figure 5(a), Chee et el. fabricated the $\mathrm{MoS}_{2}$ transistor by using graphene as the intermediate metal layer between $\mathrm{MoS}_{2}$ and $\mathrm{Ag}$ electrode [84]. The output characteristics of the controlled $\mathrm{MoS}_{2}$ transistor without using graphene and the one with $2 \mathrm{D}$ metal/semiconductor heterojunctions under different gate bias are presented in Figure 5(b), clearly demonstrating that the contact resistance of the latter device is reduced to $1.4 \%$ of the controlled one [84]. Apart from graphene, there are many metallic 2D materials such as $\mathrm{VS}_{2}, \mathrm{VSe}_{2}, \mathrm{NbS}_{2}$ the 1T or 1T' phase TMDs mentioned in Section 4.1. Ji et al. transferred the thin layer $\mathrm{VS}_{2}(<10 \mathrm{~nm})$ produced by the CVD method onto $\mathrm{MoS}_{2}$, and the source-drain current under the same bias voltage was increased by 4 times in comparison with that of the $\mathrm{MoS}_{2}$ device with the traditional $\mathrm{Ni} / \mathrm{Au}$ electrode. Compared to $1 \mathrm{~T}-\mathrm{MoS}_{2}$ or $1 \mathrm{~T}^{\prime}-\mathrm{MoTe}_{2}, 1 \mathrm{~T}-\mathrm{VS}_{2}$ exhibits stronger thermal stability, and its performance does not decline even after a few months [86].

Compared to the above transferring method, 2D semiconductor/metal heterojunctions grown directly by chemical vapor deposition have higher interface quality. Recently, Leong et al. have directly grown the lateral $\mathrm{MoS}_{2} / \mathrm{VS}_{2}$ heterostructure by CVD method. As shown in Figure 5(d), the lateral heterostructure shows good contact characteristics. The Schottky barrier height of the lateral heterostructure is only $30 \mathrm{meV}$, while the Schottky barrier height of the $\mathrm{MoS}_{2}-\mathrm{Ni}$ contact is as high as $163 \mathrm{meV}$, and the field effect mobility is increased by 6 times [87]. Such clean vdW interface can greatly suppress the interface dipole and metal-induced interstitial states. Further, by selectively forming nucleation points on semiconductor-TMDs, the growth of metallic-TMDs can be precisely controlled to form the van der Waals heterostructure arrays, as shown in Figure 5(e). In particular, $\mathrm{WSe}_{2}$ is used as the channel material, and the adjacent $\mathrm{VSe}_{2}$ layers are used as the source and drain electrodes respectively, so that $2 \mathrm{D}$ semiconductor/metal heterojunctions server as the contact. The output curve shows a satisfactory Ohmic contact, as shown in Figure 4(f). The calculated mobility is as high as $137 \mathrm{~cm}^{2} \mathrm{~V}^{-1} \mathrm{~S}^{-1}$, which is one of the highest values of $\mathrm{WSe}_{2}$ reported so far [88].

\section{Conclusion and Outlook}

In conclusion, realizing Ohmic contact is essential to achieve good device performance. Work function mismatch and Fermi level pinning are the main factors that hinder the formation of Ohmic contact. By comparing the barrier heights between different metals and 2D materials, we summarized the most suitable metals for different 2D materials including graphene, commonly used TMDs and BP. However, due to the existence of Fermi level pinning, the influence of the metal work function is weakened. We then mainly provided the mathematical representation of Fermi level pinning and presented two main methods namely intercalating an ultrathin insulating layer and transferring electrodes to reduce the influence of Fermi level pinning. Finally, we elaborated on 2D metal/semiconductor contacts including homojunction and heterojunction to reduce the contact resistance.

In addition, the use of the emerging two-dimensional materials such as silicene, germanene and borobenzene as electrodes to realize ohmic contact has also been reported recently. Yang et al. constructed Ge and MoSSe contacts on different surfaces by taking advantage of the lattice matching between Ge and MoSSe, and thus transformed the Schotty contact into ohmic contact via chaning the tensile strain [89]. Also, Zhao et al. 
systematically studied the interface properties of $\beta_{12}$ phase borophene and commonly used two-dimensional materials, and found that it can form ohmic contacts with most group V-enes (such as BP) [90].

One important area for future research and development of nanofabrication techniques is the formation of edg e contacts. Using plasma etching in combination with photoresist mask to expose the complete boundary of twodimensional materials such as graphene, and then depositing electrodes to form edge contacts. Such edge contact $\mathrm{s}$ offer the advantages of strong orbital overlap and no current crowding [33,34]. However, due to the complexity of the process, it is currently challenging to achieve pure edge contact with standard lithography technology, bec ause the photoresist will inevitably form a trapezoidal slope during the process of revealing and hiding, resulting in the formation of short and small top contact between electrodes and materials. Therefore, it is necessary to ove rcome this limitation and use more accurate methods to make edge ohmic contacts (for example, using hBN packaging [91], etc.), which requires more exploration in the future.

Although great strides have been made on how to reduce the contact resistance of 2D devices, there are still some challenges that need to be overcome. First of all, the Fermi level pinning effect is widespread in the contact between metal and 2D semiconductor, but its root cause has not been clearly explained so far. For specific materials, the role of metal interstitial states and interface dipoles can be explained as energy level pinning. But there is still no specific theoretical explanation. Secondly, for traditional semiconductor materials, Ohmic contact is mainly and effectively achieved through high doping. However, the reliable and controllable doping of 2D materials is limited, which needs further investigation. Finally, 2D metal materials are an effective means to achieve Ohmic contact. However, compared with 2D semiconductor materials, the number of 2D metal materials is very limited. Therefore, new methods for the preparation of $2 \mathrm{D}$ metal materials need to be further explored.

\section{Acknowledgements}

This work is partially supported by the National Nature Science Foundation under Grants 62074070 and 1170 4159, the Natural Science Foundation of Jiangsu Province, China under Grant BK20170167, the Fundamental R esearch Funds for the Central Universities of China under Grants JUSRP221015, the 111 Project under Grant B1 2018, as well as by the Australian Research Council (ARC).

\section{References}

[1] R. Schaller R 1997 Moore's law: past, present and future IEEE spectrum 34 52-59

[2] D.J. Frank, R.H. Dennerd, E. Nowak 2001 Device scaling limits of Si MOSFETs and their application dependencies Proceedings of the IEEE $\mathbf{8 9}$ 259-288

[3] G.E. Moore 1965 Cramming more components onto integrated circuits Proceedings of the IEEE 86 114-117

[4] G. Yeric 2015 Moore's Law at 50; Are we planning for retirement? IEEE International Electron Devices Meeting 2015 1.1.1-1.1.8

[5] M. Chhowalla, D. Jena, H. Zhang 2016 Two-dimênsional semiconductors for transistors Nat Rec Mater 116052 [6] Y. Gong, G. Shi, Z. Zhang, W. Zhou, J. Jung, W. Gao, L. Ma, Y. Yang, S. Yang, G. You, R. Vajtai, Q. Xu, A.H. MacDonald, B.I. Yakobson, J. Lou, Z. Liu, P.M. Ajayan 2014 Direct chemical conversion of graphene to boron- and nitrogen- and carbon-containing atomic layers Nat Commun 53193

[7] K.S. Novoselov, A.K. Geim, S.V. Morozov 2014 Electric Field Effect in Atomically Thin Carbon Films sicence 306 666-669

[8] A. Rai, H. Movva, A. Roy, D. Taneja, S. Chowdhury, S. Banerjee 2018 Progress in Contact, Doping and Mobility Engineering of MoS2: An Atomically Thin 2D Semiconductor Crystals 8

[9] Y. Pan, J. Gu, H. Tang, X. Zhang, J. Li, B. Shi, J. Yang, H. Zhang, J. Yan, S. Liu, H. Hu, M. Wu, J. Lu 2019 Reexamination of the Schottky Barrier Heights in Monolayer MoS2 Field-Effect Transistors ACS Applied Nano Materials 2 4717-4726

[10] N. Haiyan, j. Jie 2019 Soft hydrogen plasma induced phase transition in monolayer and fewlayer MoTe2 Nanotechnology 30034004

[11] H. Tang, B. Shi, Y. Pan, J. Li, X. Zhang, J. Yan, S. Liu, J. Yang, L. Xu, J. Yang, M. Wu, J. Lu 2019 Schottky Contact in Monolayer $\mathrm{WS}_{2}$ Field-Effect Transistors Advanced Theory and Simulations 2

[12] X. Zhang, H. Nan, S. Xiao, X. Wan, X. Gu, A. Du, Z. Ni, K.K. Ostrikov 2019 Transition metal dichalcogenides bilayer single crystals by reverse-flow chemical vapor epitaxy Nat Commun 10598

[13] H. Nan, X. Wang, J. Jiang, K. Ostrikov, Z. Ni, X. Gu, S. Xiao 2021 Effect of the surface oxide layer on the stability of black phosphorus Applied Surface Science $\mathbf{5 3 7}$

[14] R. Quhe, Y. Wang, M. Ye, Q. Zhang, J. Yang, P. Lu, M. Lei, J. Lu 2017 Black phosphorus transistors with van der Waals-type electrical contacts Nanoscale 9 14047-14057

[15] P. Ajayan, P. Kim, K. Banerjee 2016 Two-dimensional van der Waals materials Physics Today 69 38-44 
[16] R. Xu, H. Jang, M.H. Lee, D. Amanov, Y. Cho, H. Kim, S. Park, H.J. Shin, D. Ham 2019 Vertical MoS2 DoubleLayer Memristor with Electrochemical Metallization as an Atomic-Scale Synapse with Switching Thresholds Approaching $100 \mathrm{mV}$ Nano Lett 19 2411-2417

[17] Y. Zhu, Y. Li, G. Arefe, R.A. Burke, C. Tan, Y. Hao, X. Liu, X. Liu, W.J. Yoo, M. Dubey, Q. Lin, J.C. Hone 2018 Monolayer Molybdenum Disulfide Transistors with Single-Atom-Thick Gates Nano Lett 18 3807-3813

[18] J. Kang, W. Cao, X. Xie, D. Sarkar, W. Liu, K. Banerjee, Micro- and Nanotechnology Sensors, Systems, and Applications VI 2014.

[19] F. Schwierz, J. Pezoldt, R. Granzner 2015 Two-dimensional materials and their prospects in transistor electronics Nanoscale 7 8261-8283

[20] D. Akinwande, N. Petrone, J. Hone 2014 Two-dimensional flexible nanoelectronics Nat Commun 55678

[21] T. ROy, M. Tosun, J.S. Kang 2014 Field-Effect Transistors Built from All Two-Dimensional Material Components ACS Nano 8 6259-6264

[22] J.D. Mehew, S. Unal, E. Torres Alonso, G.F. Jones, S. Fadhil Ramadhan, M.F. Craciun, S. Russo 2017 Fast and Highly Sensitive Ionic-Polymer-Gated WS2 -Graphene Photodetectors Adv Mater 29

[23] X. Liu, D. Qu, R. J 2016 P-type polar transition of chemically doped multilayer MoS2 transistor Advanced Materials 28 2345-2351

[24] C.H. Liu, G. Clark, T. Fryett, S. Wu, J. Zheng, F. Hatami, X. Xu, A. Majumdar 2017 Nanocavity Integrated van der Waals Heterostructure Light-Emitting Tunneling Diode Nano Lett 17 200-205

[25] S.M. Sze, D.C. Mattis 1970 Physics of Semiconductor Devices Physics Today 23.6 75-75

[26] D.S. Schulman, A.J. Arnold, S. Das 2018 Contact engineering for 2D materials and devices Chem Soc Rev $473037-$ 3058

[27] W. Luo, M. Zhu, G. Peng, X. Zheng, F. Miao, S. Bai, X.-A. Zhang, S. Qin 2018 Carrier Modulation of Ambipolar Few-Layer MoTe2 Transistors by MgO Surface Charge Transfer Doping Advanced Functional Materials 28 [28] Y. Sheng, L. Zhang, F. Li, X. Chen, Z. Xie, H. Nan, Z. Xu, D.W. Zhang, J. Chen, Y. Pu, S. Xiao, W. Bao 2021 A novel contact engineering method for transistors based on two-dimensional materials Journal of Materials Science \& Technology 69 15-19

[29] A. Avsar, K. Marinov, E.G. Marin, G. Iannaccone, K. Watanabe, T. Taniguchi, G. Fiori, A. Kis 2018 Reconfigurable Diodes Based on Vertical WSe2 Transistors with van der Waals Bonded Contacts Adv Mater 30 e1707200

[30] F. Giubileo, A. Di Bartolomeo 2017 The role of contact resistance in graphene field-effect devices Progress in Surface Science 92 143-175

[31] K.K. Ng, W.T. Lynch 1987 The Impact of Intrinsic Series Resistance on MOSFET Scaling IEEE transactions on eletron devices 34 503-511

[32] T.H. Ning, Y. Taur 1998 Fundamentals of Modern VLSI Devices Cambrigde University Press

[33] Y. Chai, R. Ionescu, S. Su, R. Lake, M. Ozkan, C.S. Ozkan 2016 Making one-dimensional electrical contacts to molybdenum disulfide-based heterostructures through plasmâ etching physica status solidi (a) 213 1358-1364 [34] D.W. Yue, C.H. Ra, X.C. Liu, D.Y. Lee, W.J. Yoo 2015 Edge contacts of graphene formed by using a controlled plasma treatment Nanoscale 7 825-831

[35] M. Shaygan, M. Otto, A.A. Sagade 2017 Low resistive edge contacts to CVD-grown graphene using a CMOS compatible meta Annalen der Physik 5291600410

[36] X. Cui, G.H. Lee, Y.D. Kim, G. Arefe, P.Y. Huang, C.H. Lee, D.A. Chenet, X. Zhang, L. Wang, F. Ye, F. Pizzocchero, B.S. Jessen, K. Watanabe, T. Taniguchi, D.A. Muller, T. Low, P. Kim, J. Hone 2015 Multi-terminal transport measurements of MoS2 using a van der Waals heterostructure device platform Nat Nanotechnol 10 534-540 [37] R. Kappera, D. Voiry, S.E. Yalcin, B. Branch, G. Gupta, A.D. Mohite, M. Chhowalla 2014 Phase-engineered lowresistance contacts for ultrathin MoS2 transistors Nat Mater 13 1128-1134

[38] J. Zhu, Z. Wang, H. Yu, N. Li, J. Zhang, J. Meng, M. Liao, J. Zhao, X. Lu, L. Du, R. Yang, D. Shi, Y. Jiang, G. Zhang 2017 Argon Plasma Induced Phase Transition in Monolayer MoS2 J Am Chem Soc 139 10216-10219

[39] H. Nan, R. Zhou, X. Gu, S. Xiao, K. Ken Ostrikov 2019 Recent advances in plasma modification of 2D transition metal dichalcogenides Nanoscale 11 19202-19213

[40] Y. Liu, J. Guo, E. Zhu, L. Liao, S.J. Lee, M. Ding, I. Shakir, V. Gambin, Y. Huang, X. Duan 2018 Approaching the Schottky-Mott limit in van der Waals metal-semiconductor junctions Nature 557 696-700

[41] Y. Zheng, J. Gao, C. Han, W. Chen 2021 Ohmic Contact Engineering for Two-Dimensional Materials Cell Reports Physical Science 2

[42] N.F. Mott 1939 The theory of crystal rectifiers Proceedings of the Royal Society of London. Series A. Mathematical and Physical Sciences 171 27-38

[43] R.T. Tung 2014 The physics and chemistry of the Schottky barrier height Applied Physics Reviews 1

[44] O. Balci, C. Kocabas 2012 Rapid thermal annealing of graphene-metal contact Applied Physics Letters 101

[45] A. Gahoi, S. Wagner, A. Bablich, S. Kataria, V. Passi, M.C. Lemme 2016 Contact resistance study of various metal electrodes with CVD graphene Solid-State Electronics 125 234-239

[46] H.Y. Park, W.S. Jung, D.H. Kang, J. Jeon, G. Yoo, Y. Park, J. Lee, Y.H. Jang, J. Lee, S. Park, H.Y. Yu, B. Shin, S. Lee, J.H. Park 2016 Extremely Low Contact Resistance on Graphene through n-Type Doping and Edge Contact Design AdvMater 28 864-870 
[47] M. Abraham, S.E. Mohney 2017 Annealed Ag contacts to MoS2 field-effect transistors Journal of Applied Physics 122

[48] C. Maurel, F. Ajustron, R. Péchou, G. Seine, R. Coratger 2006 Electrical behavior of the Au/MoS2 interface studied by light emission induced by scanning tunneling microscopy Surface Science $\mathbf{6 0 0} 442-447$

[49] J. Kang, W. Liu, D. Sarkar 2014 Computational Study of Metal Contacts to Monolayer Transition-Metal Dichalcogenide Semiconductors Physical Review X 4031005

[50] J. Kang, W. Liu, K. Banerjee 2014 High-performance MoS2 transistors with low-resistance molybdenum contacts Applied Physics Letters 104

[51] Y. Wang, J.C. Kim, R.J. Wu, J. Martinez, X. Song, J. Yang, F. Zhao, A. Mkhoyan, H.Y. Jeong, M. Chhowalla 2019 Van der Waals contacts between three-dimensional metals and two-dimensional semiconductors Nature $\mathbf{5 6 8} 70-74$ [52] C.H. Wang, J.A.C. Incorvia, C.J. McClellan, A.C. Yu, M.J. Mleczko, E. Pop, H.P. Wong 2018 Unipolar n-Type Black Phosphorus Transistors with Low Work Function Contacts Nano Lett 18 2822-2827

[53] Z.P. Ling, S. Sakar, S. Mathew, J.T. Zhu, K. Gopinadhan, T. Venkatesan, K.W. Ang 2015 Black Phosphorus Transistors with Near Band Edge Contact Schottky Barrier Sci Rep 518000

[54] M.V. Kamalakar, B.N. Madhushankar, A. Dankert, S.P. Dash 2015 Low Schottky barrier black phosphorus fieldeffect devices with ferromagnetic tunnel contacts Small 11 2209-2216

[55] H.-M. Chang, K.-L. Fan, A. Charnas, P.D. Ye, Y.-M. Lin, C.-I. Wu, C.-H. Wu 2018 Experimental analysis of the Schottky barrier height of metal contacts in black phosphorus field-effect transistors Journal of Physics D: Applied Physics $\mathbf{5 1}$

[56] Y. Ma, C. Shen, A. Zhang, L. Chen, Y. Liu, J. Chen, Q. Liu, Z. Li, M.R. Amer, T. Nilges, A.N. Abbas, C. Zhou 2017 Black Phosphorus Field-Effect Transistors with Work Function Tunable Contacts ACS Nano 11 7126-7133 [57] D.J. Perello, S.H. Chae, S. Song, Y.H. Lee 2015 High-performance n-type black phosphorus transistors with type control via thickness and contact-metal engineering Nat Commun 67809

[58] Y. Deng, N.J. Conrad, Z. Luo 2014 Towards High-Performance Two-Dimensional Black Phosphorus Optoelectronic Devices: the Role of Metal Contacts IEEE International Electron Devices Meeting 2014 5.2.1-5.2.4

[59] K. Sotthewes, R. van Bremen, E. Dollekamp, T. Boulogne, K. Nowakowski, D. Kas, H.J.W. Zandvliet, P. Bampoulis 2019 Universal Fermi-Level Pinning in Transition-Metal Dichalcogenides $J$ Phys Chem C Nanomater Interfaces 123 5411-5420

[60] C. Gong, L. Colombo, R.M. Wallace, K. Cho 2014 The unusual mechanism of partial Fermi level pinning at metalMoS2 interfaces Nano Lett 14 1714-1720

[61] Y. Guo, D. Liu, J. Robertson 2015 Chalcogen vacancies in monolayer transition metal dichalcogenides and Fermi level pinning at contacts Applied Physics Letters 106

[62] D. Liu, Y. Guo, L. Fang, J. Robertson 2013 Sulfur vacancies in monolayer MoS2 and its electrical contacts Applied Physics Letters 103

[63] C. Kim, I. Moon, D. Lee, M.S. Choi, F. Ahmed, S. Nam, Y. Cho, H.J. Shin, S. Park, W.J. Yoo 2017 Fermi Level Pinning at Electrical Metal Contacts of Monolayer Molybdenum Dichalcogenides ACS Nano 11 1588-1596

[64] Y. Liu, P. Stradins, S.H. Wei 2016 Van der Waals metal-semiconductor junction: Weak Fermi level pinning enables effective tuning of Schottky barrier Science advances 2 e1600069

[65] Y. Guo, D. Liu, J. Robertson 2015 3D Behavior of Schottky Barriers of 2D Transition-Metal Dichalcogenides ACS Apple Mater Interfaces 7 25709-25715

[66] J.R. Chen, P.M. Odenthal, A.G. Swartz, G.C. Floyd, H. Wen, K.Y. Luo, R.K. Kawakami 2013 Control of Schottky barriers in single layer MoS2 transistors with ferromagnetic contacts Nano Lett 13 3106-3110

[67] A. Dankert, L. Langouche, M.V. Kamalakar 2014 High-Performance Molybdenum Disulfide Field-Effect Transistors with Spin Tunnel Contacts ACS Nano 8 476-482

[68] G.S. Kim, S.H. Kim, J. Park, K.H. Han, J. Kim, H.Y. Yu 2018 Schottky Barrier Height Engineering for Electrical Contacts of Multilayered MoS2 Transistors with Reduction of Metal-Induced Gap States ACS Nano 12 6292-6300 [69] S. Lee, A. Tang, S. Aloni, H.S. Wong 2016 Statistical Study on the Schottky Barrier Reduction of Tunneling Contacts to CVD Synthesized MoS2 Nano Lett 16 276-281

[70] J. Wang, Q. Yao, C.W. Huang, X. Zou, L. Liao, S. Chen, Z. Fan, K. Zhang, W. Wu, X. Xiao, C. Jiang, W.W. Wu 2016 High Mobility MoS2 Transistor with Low Schottky Barrier Contact by Using Atomic Thick h-BN as a Tunneling Layer Adv Mater 28 8302-8308

[71] X. Cui, E.M. Shih, L.A. Jauregui, S.H. Chae, Y.D. Kim, B. Li, D. Seo, K. Pistunova, J. Yin, J.H. Park, H.J. Choi, Y.H. Lee, K. Watanabe, T. Taniguchi, P. Kim, C.R. Dean, J.C. Hone 2017 Low-Temperature Ohmic Contact to Monolayer MoS2 by van der Waals Bonded Co/h-BN Electrodes Nano Lett 17 4781-4786

[72] K.-A. Min, J. Park, R.M. Wallace, K. Cho, S. Hong 2016 Reduction of Fermi level pinning at Au-MoS ${ }_{2}$ interfaces by atomic passivation on Au surface 2 D Materials 4

[73] C.D. English, G. Shine, D.V. E 2016 Improved Contacts to MoS2 Transistors by Ultra-High Vacuum Metal Deposition Nano letters 16 3824-3830

[74] A. Matković, A. Petritz, G. Schider, M. Krammer, M. Kratzer, E. Karner-Petritz, A. Fian, H. Gold, M. Gärtner, A. Terfort, C. Teichert, E. Zojer, K. Zojer, B. Stadlober 2020 Interfacial Band Engineering of MoS2/Gold Interfaces Using Pyrimidine-Containing Self-Assembled Monolayers: Toward Contact-Resistance-Free Bottom-Contacts Advanced Electronic Materials 6 
[75] S. Liu, J. Li, B. Shi, X. Zhang, Y. Pan, M. Ye, R. Quhe, Y. Wang, H. Zhang, J. Yan, L. Xu, Y. Guo, F. Pan, J. Lu 2018 Gate-tunable interfacial properties of in-plane ML MX2 1T'-2H heterojunctions Journal of Materials Chemistry C 6 5651-5661

[76] Z.Q. Fan, X.W. Jiang, J. Chen, J.W. Luo 2018 Improving Performances of In-Plane Transition-Metal Dichalcogenide Schottky Barrier Field-Effect Transistors ACS Appl Mater Interfaces 10 19271-19277 [77] D.H. Keum, S. Cho, J.H. Kim, D.-H. Choe, H.-J. Sung, M. Kan, H. Kang, J.-Y. Hwang, S.W. Kim, H. Yang, K.J. Chang, Y.H. Lee 2015 Bandgap opening in few-layered monoclinic MoTe2 Nature Physics 11 482-486 [78] J.H. Sung, H. Heo, S. Si, Y.H. Kim, H.R. Noh, K. Song, J. Kim, C.S. Lee, S.Y. Seo, D.H. Kim, H.K. Kim, H.W. Yeom, T.H. Kim, S.Y. Choi, J.S. Kim, M.H. Jo 2017 Coplanar semiconductor-metal circuitry defined on few-layer MoTe2 via polymorphic heteroepitaxy Nat Nanotechnol 12 1064-1070 [79] Y. Ma, B. Liu, A. Zhang 2015 Reversible Semiconducting-to-Metallic Phase Transition in Chemical Vapor Deposition Grown Monolayer WSe2 and Applications for Devices ACS Nano 9 7383-7391 [80] C.S. Lee, S.J. Oh, H. Heo, S.Y. Seo, J. Kim, Y.H. Kim, D. Kim, O.F. Ngome Okello, H. Shin, J.H. Sung, S.Y. Choi, J.S. Kim, J.K. Kim, M.H. Jo 2019 Epitaxial van der Waals Contacts between Transition-Metal Dichalcogenide Monolayer Polymorphs Nano Lett 19 1814-1820 [81] S. Cho, S. Kim, J.H. Kim 2015 Phase patterning for ohmic homojunction contact in MoTe2 Science 349 625-628 [82] L. Zhang, H. Nan, X. Zhang, Q. Liang, A. Du, Z. Ni, X. Gu, K.K. Ostrikov, S. Xiao 20202 D atomic crystal molecular superlattices by soft plasma intercalation Nat Commun 115960 [83] T. Das, E. Yang, J.E. Seo, J.H. Kim, E. Park, M. Kim, D. Seo, J.Y. Kwak, J. Chang 2021 Doping-Free All PtSe2 Transistor via Thickness-Modulated Phase Transition ACS Appl Mater Interfaces 13 1861-1871 [84] S.S. Chee, D. Seo, H. Kim, H. Jang, S. Lee, S.P. Moon, K.H. Lee, S.W. Kim, H. Choi, M.H. Ham 2019 Lowering the Schottky Barrier Height by Graphene/Ag Electrodes for High-Mobility MoS2 Field-Effect Transistors Adv Mater 31 e1804422

[85] Y. Sata, R. Moriya, S. Masubuchi 2017 N- and p-type carrier injections into WSe2 with van der Waals contacts of two-dimensional materials Japanese Journal of Applied Physics 5604 CK09

[86] Q. Ji, C. Li, J. Wang 2017 Metallic vanadium disulfide nanosheets as a platform material for multifunctional electrode applications Nano letters 17 4908-4916

[87] W.S. Leong, Q. Ji, N. Mao, Y. Han, H. Wang, A.J. Goodman, A. Vignon, C. Su, Y. Guo, P.C. Shen, Z. Gao, D.A. Muller, W.A. Tisdale, J. Kong 2018 Synthetic Lateral Metal-Semiconductor Heterostructures of Transition Metal Disulfides J Am Chem Soc 140 12354-12358

[88] J. Li, X. Yang, Y. Liu, B. Huang, R. Wu, Z. Zhang, B. Zhao, H. Ma, W. Dang, Z. Wei, K. Wang, Z. Lin, X. Yan, M. Sun, B. Li, X. Pan, J. Luo, G. Zhang, Y. Liu, Y. Huang, X. Duan, X. Duan 2020 General synthesis of two-dimensional van der Waals heterostructure arrays Nature 579 368-374

[89] N. Zhao, U. Schwingenschlogl 2020 Transition from Schottky to Ohmic contacts in Janus MoSSe/germanene heterostructures Nanoscale 12 11448-11454

[90] J. Yang, R. Quhe, S. Feng, Q. Zhang, M. Lei, J. Lu 2017/Interfacial properties of borophene contacts with twodimensional semiconductors Phys Chem Chem Phys 19 23982-23989

[91] B. Karpiak, A. Dankert, A.W. Cummings, S.R. Power, S. Roche, S.P. Dash 2017 1D ferromagnetic edge contacts to 2D graphene/h-BN heterostructures 2 D Materials 5 
(a) $-\underline{E_{v a c}}$

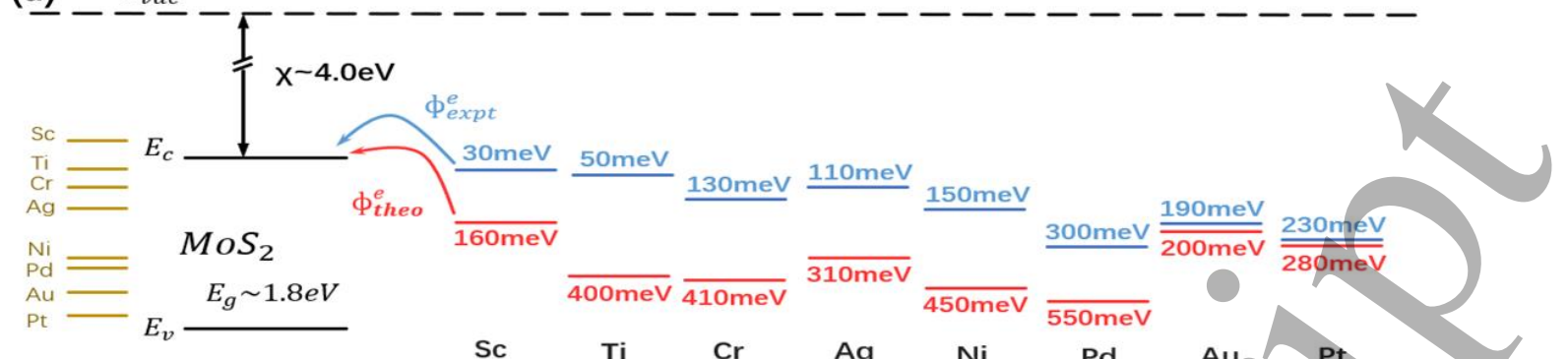

(b)
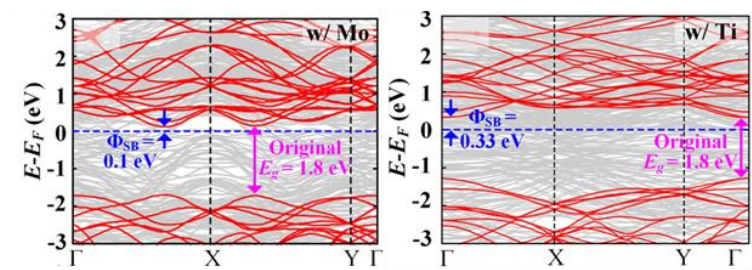

(c)

(d)

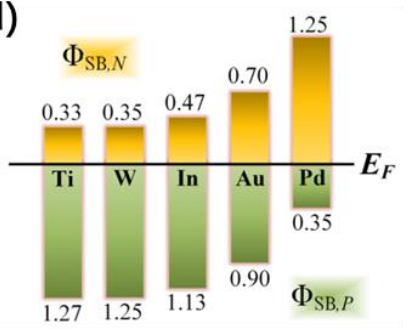

(e)

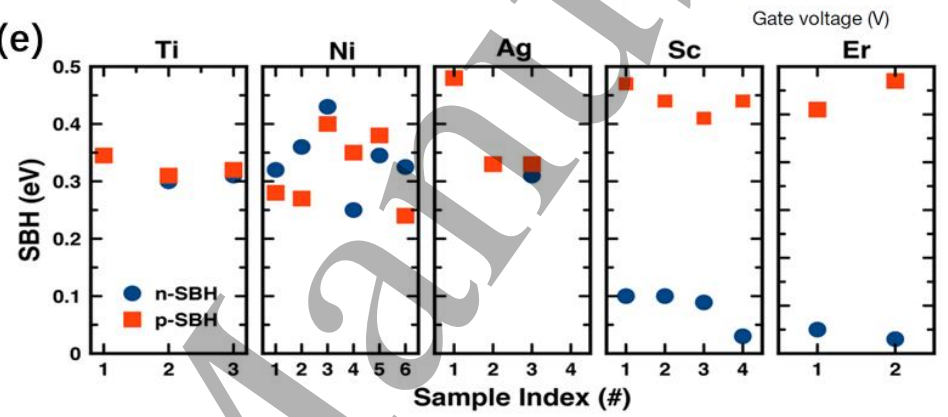

Figure 1: TMDCs and BP are in contact with metals with different work functions. (a) The energy band of MoS 2 and the work function arrangement of commonly used metals (left side), and the n-type Schottky barrier height where different metals contact with $\mathrm{MoS}_{2}$ (right side). The red part is the electronic Schottky barrier height obtained through theoretical calculation [9], and the blue part is the Schottky barrier height extracted in the actual experiment [11, 47, 48]. (b) Band structure of Mo-MoS $\mathrm{S}_{2}$ and Ti-MoS 2 [50]; (c) Van der Waals contact between In and $\mathrm{MoS}_{2}$ [51]; (d) Barrier heights of five different metals in contact with $\mathrm{WSe}_{2}$ [49]; (e) The Schottky barrier heights of five commonly used metals in contact with BP (N-SBH, p-SBH)[52]. 
(a)

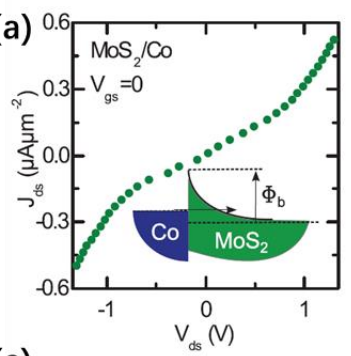

(c)
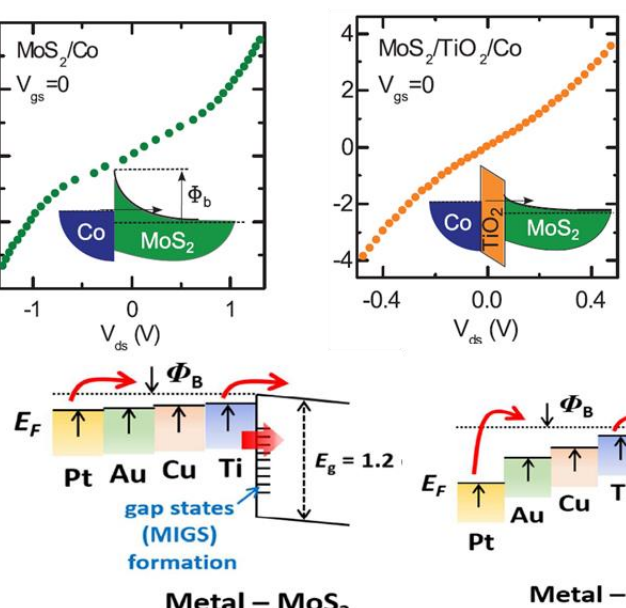

(b)

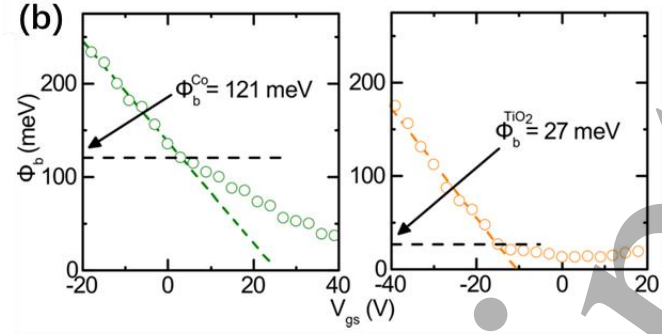

(d)

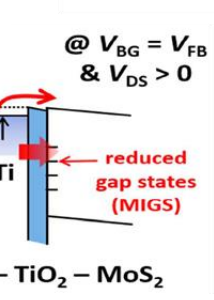

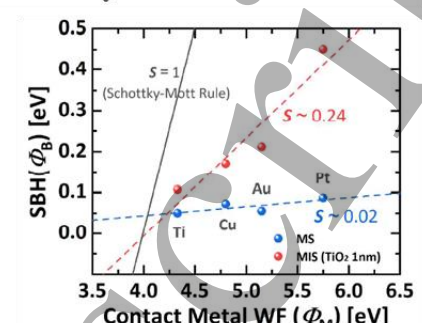

(e)
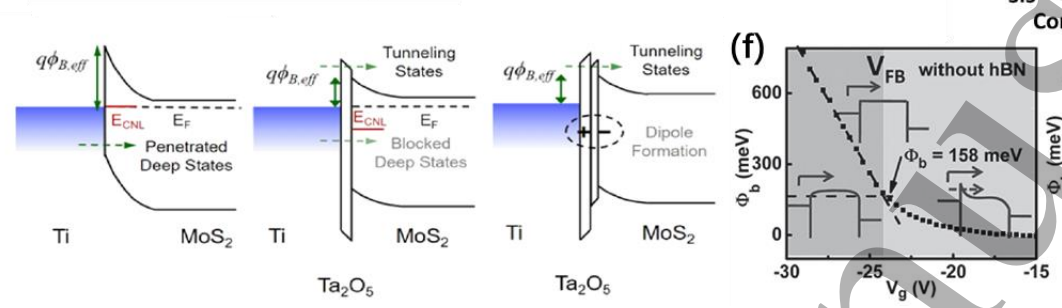
Contact Metal WF $\left(\Phi_{\mathrm{M}}\right)[\mathrm{eV}]$

Figure 2: Oxide intercalation weakens the Fermi level pinning effect. (a) $\mathrm{MoS}_{2}$ output curve before and after oxide intercalation under zero gate voltage. (b) The height of the Schottky barrier extracted from Figure2(a)[67]. (c) Schematic diagram of the structure of MS S/D contact and MISS/D contact. (d) The height of the Schottky barrier extracted before and after $\mathrm{TiO}_{2}$ intercalation, and the fitted pinning coefficient S[68]. (e) Schematic diagram of the Fermi level of MoS 2 pinning, and two explanations for reducing the Fermi pinning effect [69]. (f) Inserting h-BN to form the MIS structure reducing the barrier height from $158 \mathrm{meV}$ to $31 \mathrm{meV}$ (the illustration is the device structure diagram) [70]. 


\section{1}

(a)
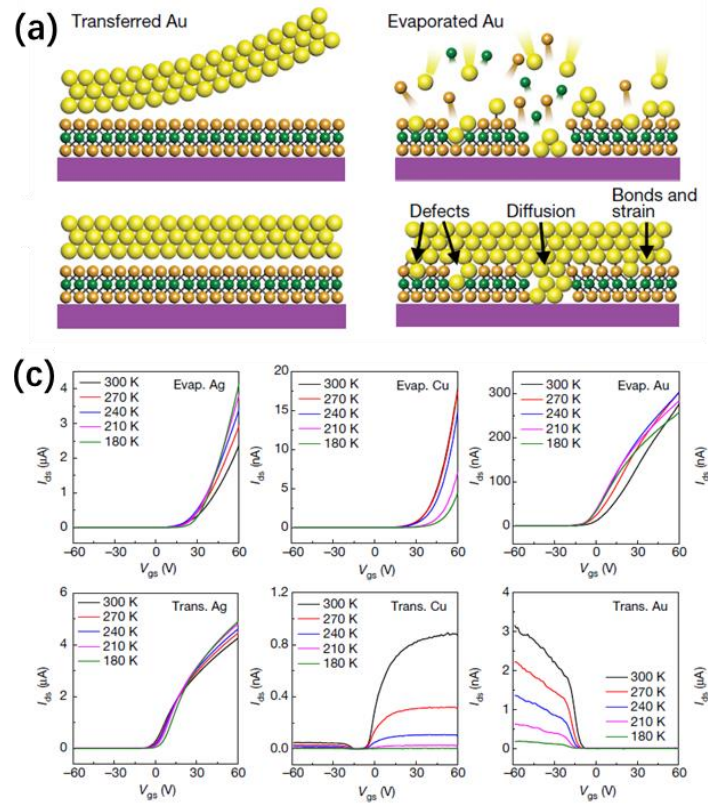

(b)
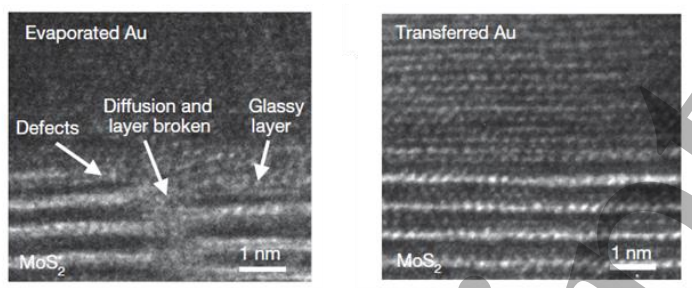

(d)
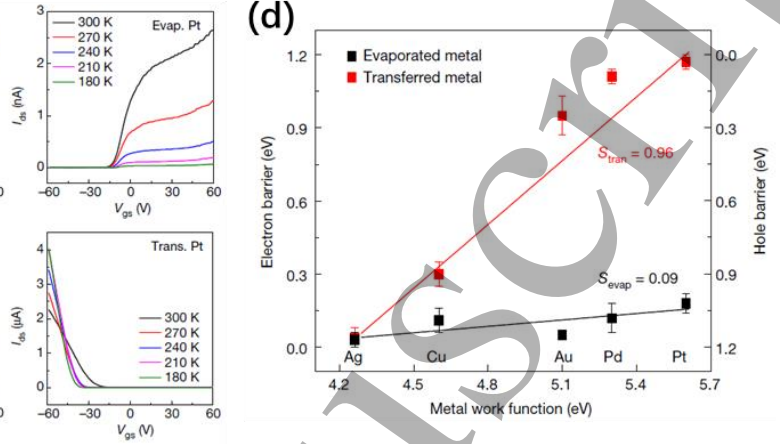

Figure 3: (a)-(b) Cross-sectional schematics and TEM images of the transferred Au electrode on top of $\mathrm{MoS}_{2}$ and conventional electron-beam-deposited $\mathrm{Au}$ electrode on top of $\mathrm{MoS}_{2}$. The transferred metal-semiconductor surface is very clean and atomically sharp, while electron beam evaporation creates considerable damage to the $\mathrm{MoS}_{2}$ surface, producing a glassy layer with apprarent defects, interface diffusion, chemical bonding and atomic disorder. (c) Transfer characteristic curves of $\mathrm{MoS}_{2}$ transistors with deposited and transferred metal electrodes. (d) Expermentally determined Schottky barrier height for different transferred metals and evaporated metals [40]. 
(a)

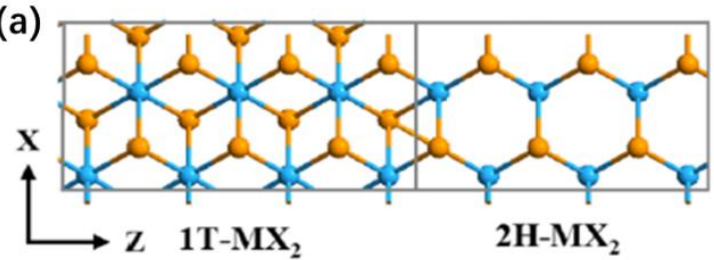

(b)

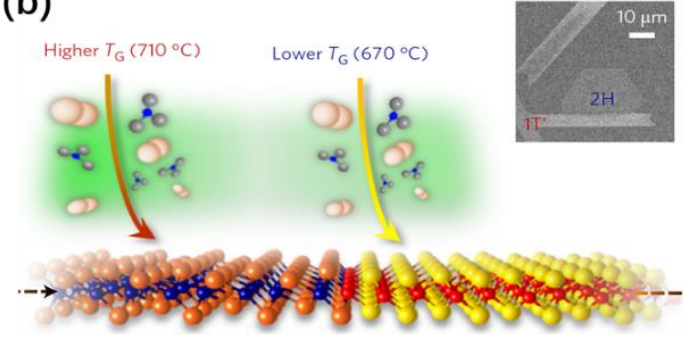

(d)

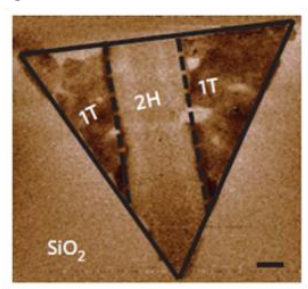

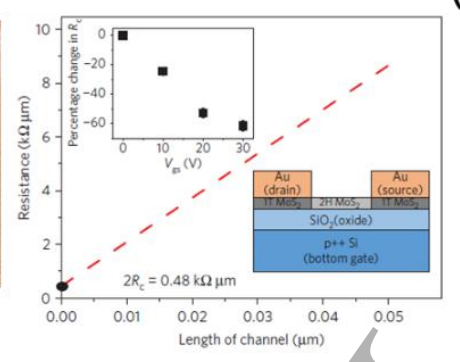

(c)

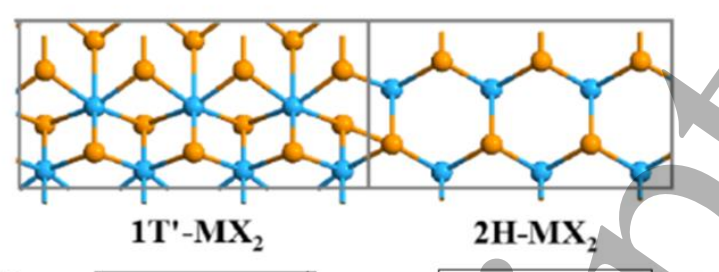

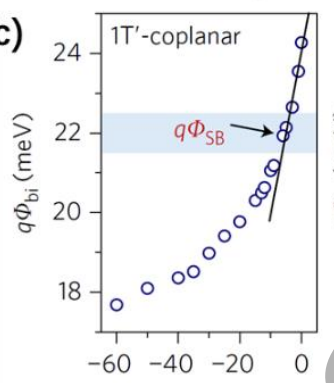

(e)

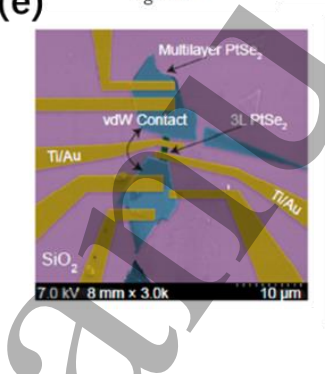

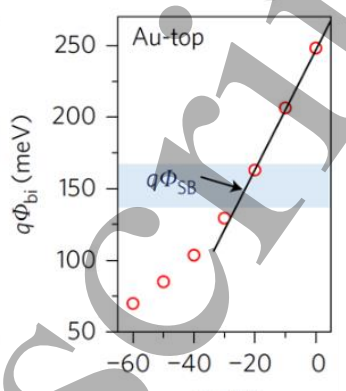

$V_{g}(V)$

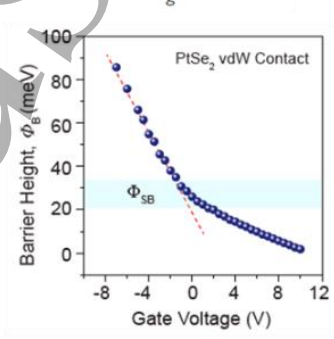

Figure 4: 2D metal and 2D semiconductor homojunction. (a) In-plane atomic connection diagrams for $1 \mathrm{~T}-\mathrm{MX}_{2}$ and $2 \mathrm{H}_{-} \mathrm{MX}_{2}$ as well as $1 \mathrm{~T}^{\prime}-\mathrm{MX}_{2}$ and $2 \mathrm{H}=\mathrm{MX}_{2}$ homojunctions[76]. (b) Schematic and SEM image of the growth of lateral homojunction consisting of $2 \mathrm{H} \mathrm{MoTe}_{2}$ and $1 \mathrm{~T} \mathrm{MoTe} 2$ by chemical vapor deposition via adjusting growth temperature[78]. (c) The extracted Schottky barrier heights for the novel contact consisting of $2 \mathrm{H} / 1 \mathrm{~T}$ ' homojunction and metal electrode as well as the traditional contact consisting of $2 \mathrm{H} \mathrm{MoTe}_{2}$ and metal electrode [78]. (d) $\mathrm{MoS}_{2}$ is transformed from $2 \mathrm{H}$ phase to $1 \mathrm{~T}$ phase through phase engineering, and the contact resistance of the corresponding transistor is calculated under zero gate bias[37]. (e) A false-color top-view SEM image of the representative few layer PtSe ${ }_{2}$ FETs and the correspongding extracted Schottky barrier height[83]. 
(a)
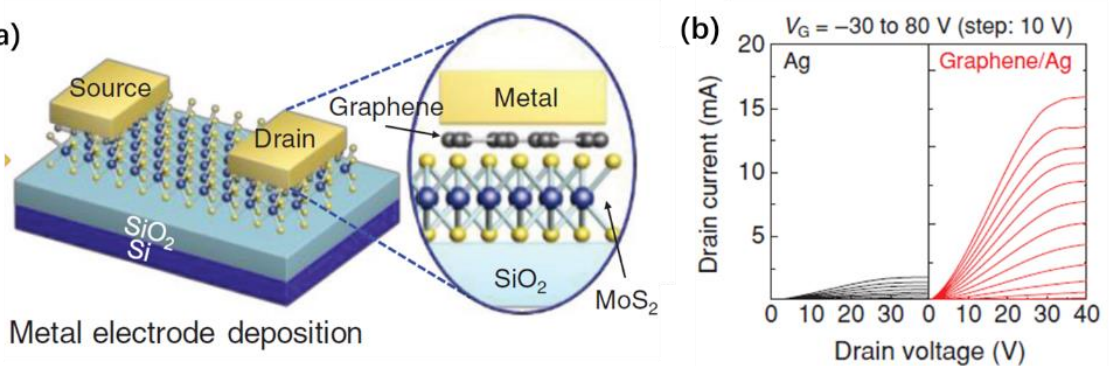

(c)

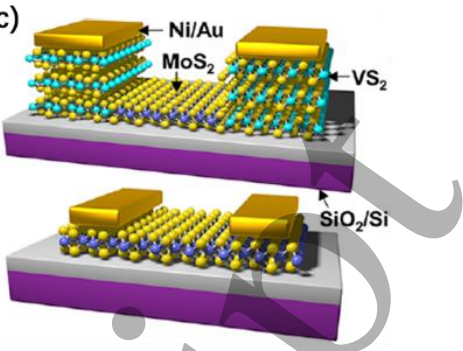

(d)

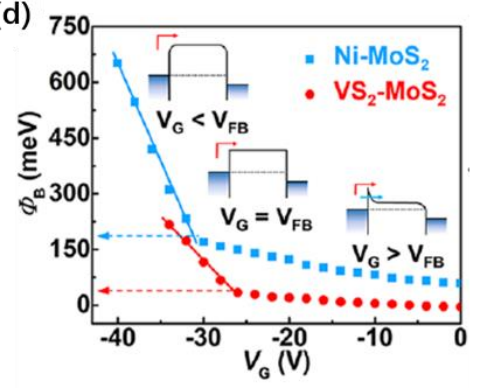

(e)

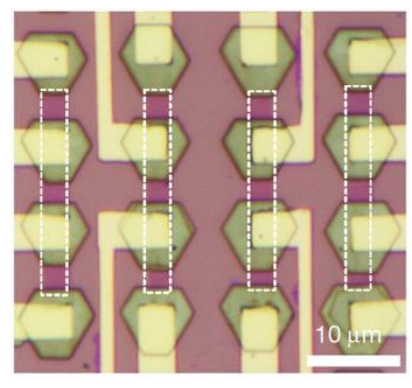

(f)

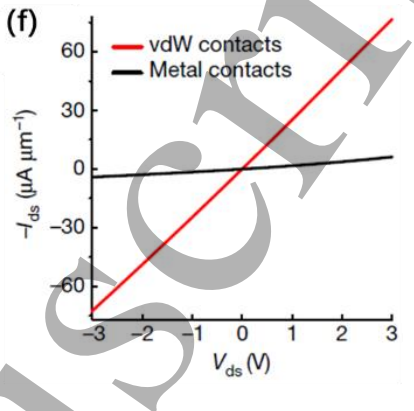

Figure 5: 2D metal and 2D semiconductor heterojunction. (a) Schematic diagram of graphene being transferred to $\mathrm{MoS}_{2}$ as an electrode. (b) Output characteristics of $\mathrm{MoS}_{2}$ FETs with Ag and graphene/Ag contacts under different gate bias voltage[84]. (c) Schematics showing monolayer $\mathrm{MoS}_{2}$ field-effect transistors (FETs) with the lateral $\mathrm{VS}_{2}$ contacts (upper) and vertical $\mathrm{Ni}$ contacts (lower). (d) Schottky barrier heights $(\Phi B)$ of $\mathrm{VS}_{2}-$ and Ni-contacted $\mathrm{MoS}_{2}$ devices, as a function of VG. Dashed arrows indicate flat-band $\Phi_{\mathrm{B}}[87]$. (e) Typical optical microscopy images of rectangular periodic arrangements of $\mathrm{VSe}_{2} / \mathrm{WSe}_{2} \mathrm{vdWH}$ arrays. (f) Comparison of output curves of van der Waals contacts and $\mathrm{WSe}_{2}$ transistors with direct deposition metal contacts [88]. 